hep-th/0507112

July, 2005

\title{
On Reductions of Noncommutative Anti-Self-Dual Yang-Mills Equations
}

\author{
Masashi Hamanaka ${ }^{1}$ \\ Graduate School of Mathematics, Nagoya University, \\ Chikusa-ku, Nagoya, 464-8602, JAPAN \\ Mathematical Institute, University of Oxford, \\ 24-29, St Giles', Oxford, OX1 3LB, UK
}

\begin{abstract}
We show that various noncommutative integrable equations can be derived from noncommutative anti-self-dual Yang-Mills equations in the split signature, which include noncommutative versions of Korteweg-de Vries, Non-Linear Schrödinger, $N$-wave, DaveyStewartson and Kadomtsev-Petviashvili equations. $U(1)$ part of gauge groups for the original Yang-Mills equations play crucial roles in noncommutative extension of MasonSparling's celebrated discussion. The present results would be strong evidences for noncommutative Ward's conjecture and imply that these noncommutative integrable equations could have the corresponding physical pictures such as reduced configurations of D0-D4 brane systems in open $\mathrm{N}=2$ string theories. Possible applications to the D-brane dynamics are also discussed.
\end{abstract}

\footnotetext{
${ }^{1}$ The author visits Oxford from 16 August, 2005 to 15 August, 2006.

E-mail: hamanaka@math.nagoya-u.ac.jp, hamanaka@maths.ox.ac.uk
} 


\section{Introduction}

Field theories on non-commutative (NC) spaces have been studied intensively for the last several years. NC gauge theories are equivalent to ordinary gauge theories in the presence of background magnetic fields and succeeded in revealing various aspects of them [1]. NC solitons especially play important roles in the study of D-brane dynamics, such as the confirmation of Sen's conjecture on tachyon condensation [2]. One of the distinguished features of NC theories is resolution of singularities. This gives rise to various new physical objects such as U(1) instantons and makes it possible to analyze singular configurations as usual [3].

NC extension of integrable equations is also one of the hot topics (For reviews, see [4.) and has been studied from various viewpoints by many authors [5]-47]. These equations imply no gauge field and $\mathrm{NC}$ extension of them perhaps might have no physical picture or no good property on integrabilities. To make matters worse, NC extension of $(1+1)$ dimensional equations introduces infinite number of time derivatives, which makes it hard to discuss or define the integrability.

Nevertheless, some of them actually possess integrable-like properties, such as the existence of infinite number of conserved quantities and exact multi-soliton solutions, and the linearizability and so on. Furthermore, a few of them can be derived from NC (anti)self-dual Yang-Mills (ASDYM) equations by suitable reductions, which is just an example of NC version [27] of Ward's conjecture [48], that is, almost all NC integrable equations are reductions of NC ASDYM equations. (For commutative discussions, see e.g. [49]52.) This makes it possible to give physical pictures into lower-dimensional integrable equations and to apply analysis of NC solitons to that of the corresponding D-branes. Origin of the integrable-like properties would be also explained from the viewpoints of $\mathrm{NC}$ twistor theory and the preserved supersymmetry in the D-brane systems. That is why confirmation of $\mathrm{NC}$ Ward conjecture is very important.

In this letter, we show that various $\mathrm{NC}$ integrable equations ${ }^{2}$ can be derived from NC ASDYM equations on $(2+2)$-dimensional flat spaces. The signature of metric is $(+,+,-,-)$ and called the split signature. In section 3, we extend Mason-Sparling's celebrated results [53] to NC spaces and derive NC Korteweg-de Vries (KdV) and NonLinear Schrödinger (NLS) equations on NC $(1+1)$-dimensional space-time. We can prove that the gauge groups in original gauge theories must be $G=G L(2, \mathbf{R})$ not $G=S L(2, \mathbf{R})$ and $G=U(2)$ not $G=S U(2)$. Hence we conclude that $U(1)$ part of the gauge groups play crucial roles as usual in NC gauge theories. In section 4, we derive $\mathrm{NC} N$-wave, DaveyStewartson (DS) and Kadomtsev-Petviashvili (KP) equations on NC $(2+1)$-dimensional

\footnotetext{
${ }^{2}$ In the present letter, integrability of $\mathrm{NC}$ equations means existence of infinite number of conserved quantities or exact multi-soliton solutions.
} 
space-time where the Lie algebras are operator algebras. The present results imply that these NC integrable equations could have actually the corresponding physical pictures such as reduced D-brane configurations of D0-D4 brane systems in open $\mathrm{N}=2$ string theories in background of $B$-fields [54]. Possible applications to the D-brane dynamics are also discussed in section 5 .

\section{NC ASDYM Equations}

NC spaces are defined by the noncommutativity of the coordinates:

$$
\left[x^{i}, x^{j}\right]=i \theta^{i j}
$$

where $\theta^{i j}$ are real constants and called the $N C$ parameters.

$\mathrm{NC}$ field theories are given by the exchange of ordinary products in the commutative field theories for the star-products and realized as deformed theories from the commutative ones.

The star-product is defined for ordinary fields on commutative spaces. For Euclidean spaces, it is explicitly given by

$$
\begin{aligned}
f \star g(x) & :=\left.\exp \left(\frac{i}{2} \theta^{i j} \partial_{i}^{\left(x^{\prime}\right)} \partial_{j}^{\left(x^{\prime \prime}\right)}\right) f\left(x^{\prime}\right) g\left(x^{\prime \prime}\right)\right|_{x^{\prime}=x^{\prime \prime}=x} \\
& =f(x) g(x)+\frac{i}{2} \theta^{i j} \partial_{i} f(x) \partial_{j} g(x)+O\left(\theta^{2}\right)
\end{aligned}
$$

where $\partial_{i}^{\left(x^{\prime}\right)}:=\partial / \partial x^{i}$ and so on. This explicit representation is known as the Moyal product [55]. The star-product has associativity: $f \star(g \star h)=(f \star g) \star h$, and returns back to the ordinary product in the commutative limit: $\theta^{i j} \rightarrow 0$. The modification of the product makes the ordinary spatial coordinate "noncommutative," that is, $\left[x^{i}, x^{j}\right]_{\star}:=$ $x^{i} \star x^{j}-x^{j} \star x^{i}=i \theta^{i j}$.

We note that the fields themselves take c-number values as usual and the differentiation and the integration for them are well-defined as usual. A nontrivial point is that NC field equations contain infinite number of derivatives in general. Hence the integrability of the equations are not so trivial as commutative cases. Nevertheless, NC ASDYM equations are integrable in some sense. (See, e.g. [11, 12, 19.)

Let us consider Yang-Mills theories on $(2+2)$-dimensional NC spaces. Here, we follow the convention in 49. NC ASDYM equation is derived from compatibility condition of the following linear system:

$$
\left(D_{w}-\zeta D_{\tilde{z}}\right) \star \Psi=0, \quad\left(D_{z}-\zeta D_{\tilde{w}}\right) \star \Psi=0,
$$


where $(z, \tilde{z}, w, \tilde{w})$ and $D_{z}, D_{w}, D_{\tilde{z}}, D_{\tilde{w}}$ denote the coordinates of the original $(2+2)$ dimensional space and covariant derivatives in the Yang-Mills theory, respectively. Explicit representations are as follows:

$$
\begin{aligned}
& \partial_{w} A_{z}-\partial_{z} A_{w}+\left[A_{w}, A_{z}\right]_{\star}=0, \quad \partial_{\tilde{w}} A_{\tilde{z}}-\partial_{\tilde{z}} A_{\tilde{w}}+\left[A_{\tilde{w}}, A_{\tilde{z}}\right]_{\star}=0, \\
& \partial_{z} A_{\tilde{z}}-\partial_{\tilde{z}} A_{z}+\partial_{\tilde{w}} A_{w}-\partial_{w} A_{\tilde{w}}+\left[A_{z}, A_{\tilde{z}}\right]_{\star}-\left[A_{w}, A_{\tilde{w}}\right]_{\star}=0 .
\end{aligned}
$$

where $A_{z}, A_{w}, A_{\tilde{z}}, A_{\tilde{w}}$ denote the gauge fields, respectively. We note that the commutator part should remain even when the gauge group is $U(1)$ because the elements of the gauge group could be operators and the gauge group could be considered to be non-abelian: $U(\infty)$. This $U(1)$ part actually plays important roles as NC gauge theories and gives rise to new physical objects [1, 3].

Taking various reduction conditions of NC ASDYM equations for, such as, choice of gauge groups, translation invariance, gauge fixings, and further constraints on gauge fields, we can get $\mathrm{NC}$ integrable equations in $(2+1)$ and $(1+1)$-dimensional spaces.

\section{$3 \quad \mathrm{NC} \mathrm{KdV}$ and NLS Equations from ASDYM}

In this section, we discuss reductions of NC ASDYM equations to $(1+1)$-dimensional space-time, including NC NLS eq., NC KdV equation, which is $\mathrm{NC}$ extension of the Mason-Sparling's result [53]. Relation to hyperCR Einstein-Weyl structure has been also discussed by Dunajski and Sparling [56]. In this section, we follow the convention in [49].

Let us consider the following NC ASDYM equation with which is dimensionally reduced to 2-dimensional space-time with the coordinate $(t, x)=(z, w+\tilde{w})$ :

$$
Q^{\prime}=0, \quad \dot{Q}+A_{w}^{\prime}+\left[A_{z}, Q\right]_{\star}=0, \quad A_{z}^{\prime}-\dot{A}_{w}+\left[A_{w}, A_{z}\right]_{\star}=0 .
$$

where $Q, A_{w}$ and $A_{z}$ are $2 \times 2$ matrices and denote the original gauge fields, and $Q^{\prime}:=$ $\partial Q / \partial x, \dot{Q}:=\partial Q / \partial t$.

- NC KdV equation

Now let us take further reduction on the gauge fields in the ASDYM equation (3.1) as follows:

$$
\begin{aligned}
& Q=\left(\begin{array}{ll}
0 & 0 \\
1 & 0
\end{array}\right), A_{w}=\left(\begin{array}{cc}
q & -1 \\
q^{\prime}+q \star q & -q
\end{array}\right), \\
& A_{z}=\left(\begin{array}{cc}
\frac{1}{2} q^{\prime \prime}+a q \star q^{\prime}+(1-a) q^{\prime} \star q & -q^{\prime} \\
\phi & -\frac{1}{2} q^{\prime \prime}-b q^{\prime} \star q-(1-b) q \star q^{\prime}
\end{array}\right),
\end{aligned}
$$


where $a$ and $b$ are constants, and $\phi$ is a differential polynomial of $q$.

The first equation of (3.1) is trivially satisfied. The second equation of (3.1) becomes

$$
\left(\begin{array}{cc}
0 & 0 \\
(a-b)\left[q^{\prime}, q\right]_{\star} & 0
\end{array}\right)=0,
$$

which leads to $a=b$. Then, the third equation of (3.1) yields

$$
\begin{aligned}
\phi= & \frac{1}{2} q^{\prime \prime \prime}+2 q^{\prime} \star q^{\prime}-\dot{q}+a\left\{q \star q, q^{\prime}\right\}_{\star}+(1-2 a) q \star q^{\prime} \star q \\
& +\frac{1}{2}\left\{q, q^{\prime \prime}\right\}_{\star}+a\left[q, q^{\prime \prime}\right]_{\star}, \\
\phi= & \frac{1}{2} q^{\prime \prime \prime}+2 q^{\prime} \star q^{\prime}-\dot{q}+a\left\{q \star q, q^{\prime}\right\}_{\star}+(1-2 a) q \star q^{\prime} \star q \\
& +\frac{1}{2}\left\{q, q^{\prime \prime}\right\}_{\star}-a\left[q, q^{\prime \prime}\right]_{\star}, \\
\phi^{\prime}= & \dot{q}^{\prime}+\dot{q} \star q+q \star \dot{q}+\{\phi, q\}_{\star}-\frac{1}{2}\left\{q^{\prime \prime}, q \star q+q^{\prime}\right\}_{\star}-a\left\{q \star q \star q, q^{\prime}\right\}_{\star} \\
& -(1-a)\left\{q, q^{\prime} \star q^{\prime}+q \star q^{\prime} \star q\right\}_{\star}-2 a q^{\prime} \star q \star q^{\prime},
\end{aligned}
$$

where $\{A, B\}_{\star}:=A \star B+B \star A$.

From Eqs. (3.3) and (3.4), we get $a\left[q, q^{\prime \prime}\right]_{\star}=0$, hence, $a=0$. (The published version shows incorrect derivation of $a=0$.)

If we take

$$
\phi=\frac{1}{4} q^{\prime \prime \prime}+\frac{1}{2} q^{\prime} \star q^{\prime}+\frac{1}{2}\left\{q, q^{\prime \prime}\right\}_{\star}+q \star q^{\prime} \star q,
$$

Eqs. (3.3) and (3.4) become a NC potential KdV equation:

$$
\dot{q}=\frac{1}{4} q^{\prime \prime \prime}+\frac{3}{2} q^{\prime} \star q^{\prime}
$$

This is derived from the NC KdV equation

$$
\dot{u}=\frac{1}{4} u^{\prime \prime \prime}+\frac{3}{4}\left(u^{\prime} \star u+u \star u^{\prime}\right)
$$

by setting $2 q^{\prime}=u$. Then Eq. (3.5) also leads to the NC potential KdV equation as follows

$$
\left(-\dot{q}+\frac{1}{4} q^{\prime \prime \prime}+\frac{3}{2} q^{\prime} \star q^{\prime}\right)^{\prime}+q \star\left(-\dot{q}+\frac{1}{4} q^{\prime \prime \prime}+\frac{3}{2} q^{\prime} \star q^{\prime}\right)+\left(-\dot{q}+\frac{1}{4} q^{\prime \prime \prime}+\frac{3}{2} q^{\prime} \star q^{\prime}\right) \star q=0 .
$$


In summary, only when $a=b=0$, we can obtain the NC KdV equation (3.8) by reduction of $\mathrm{NC}$ ASDYM equation under the reduction conditions:

$$
\begin{aligned}
& Q=\left(\begin{array}{ll}
0 & 0 \\
1 & 0
\end{array}\right), A_{w}=\left(\begin{array}{cc}
q & -1 \\
q^{\prime}+q \star q & -q
\end{array}\right), \\
& A_{z}=\left(\begin{array}{cc}
\frac{1}{2} q^{\prime \prime}+q^{\prime} \star q & -q^{\prime} \\
\frac{1}{4} q^{\prime \prime \prime}+\frac{1}{2} q^{\prime} \star q^{\prime}+\frac{1}{2}\left(q \star q^{\prime \prime}+q^{\prime \prime} \star q\right)+q \star q^{\prime} \star q & -\frac{1}{2} q^{\prime \prime}-q \star q^{\prime}
\end{array}\right),
\end{aligned}
$$

We note that the gauge group is not $S L(2, \mathbf{R})$ but $G L(2, \mathbf{R})$ on NC spaces because the matrix $A_{z}$ is not traceless. This result also reflects the importance of $U(1)$ part of the original gauge group.

- NC NLS equation

Now let us take another further reduction on the gauge fields in the ASDYM equation (3.1) as follows:

$$
\begin{aligned}
& Q=\kappa\left(\begin{array}{cc}
1 & 0 \\
0 & -1
\end{array}\right), A_{w}=\left(\begin{array}{cc}
0 & \psi \\
\tilde{\psi} & 0
\end{array}\right), \\
& A_{z}=\frac{1}{2 \kappa}\left(\begin{array}{cc}
a \psi \star \tilde{\psi}+\left(\begin{array}{c}
1-a) \tilde{\psi} \star \psi \\
-\tilde{\psi}^{\prime}
\end{array}\right. & -b \tilde{\psi} \star \psi-(1-b) \psi \star \tilde{\psi}
\end{array}\right) .
\end{aligned}
$$

These conditions automatically solve the first and the second equations in (3.1) and the third equation is reduced to

$$
\begin{aligned}
& \frac{a-1}{2 \kappa}\left(\psi^{\prime} \star \tilde{\psi}+\psi \star \tilde{\psi}^{\prime}-\tilde{\psi}^{\prime} \star \psi-\tilde{\psi} \star \psi^{\prime}\right)=0, \\
& \frac{1-b}{2 \kappa}\left(\tilde{\psi}^{\prime} \star \psi+\tilde{\psi} \star \psi^{\prime}-\psi^{\prime} \star \tilde{\psi}-\psi \star \tilde{\psi}^{\prime}\right)=0, \\
& -\dot{\psi}+\frac{1}{2 \kappa}\left(\psi^{\prime \prime}-(a+b) \psi \star \tilde{\psi} \star \psi-(1-a) \tilde{\psi} \star \psi \star \psi-(1-b) \psi \star \psi \star \tilde{\psi}\right)=0, \\
& -\dot{\tilde{\psi}}+\frac{1}{2 \kappa}\left(-\tilde{\psi}^{\prime \prime}+(a+b) \tilde{\psi} \star \psi \star \tilde{\psi}+(1-a) \tilde{\psi} \star \tilde{\psi} \star \psi+(1-b) \psi \star \tilde{\psi} \star \tilde{\psi}\right)=0 .
\end{aligned}
$$

We can also see that only when $a=b=1$, these equations are reduced to

$$
2 \kappa \dot{\psi}=\psi^{\prime \prime}-2 \psi \star \tilde{\psi} \star \psi, \quad 2 \kappa \dot{\tilde{\psi}}=-\tilde{\psi}^{\prime \prime}+2 \tilde{\psi} \star \psi \star \tilde{\psi}
$$

By taking $\kappa=i / 2, \tilde{\psi}=\bar{\psi}$, we get the NC repulsive NLS equation with $G=U(1,1)$ :

$$
i \dot{\psi}=\psi^{\prime \prime}-2 \psi \star \bar{\psi} \star \psi
$$

and by taking $\kappa=i / 2, \tilde{\psi}=-\bar{\psi}$, the NC attractive NLS equation [8] with $G=U(2)$ :

$$
i \dot{\psi}=\psi^{\prime \prime}+2 \psi \star \bar{\psi} \star \psi
$$


In summary, only when $a=b=1$, we can obtain the NC NLS equation by reduction of NC ASDYM equation under the reduction conditions:

$$
Q=\frac{i}{2}\left(\begin{array}{cc}
1 & 0 \\
0 & -1
\end{array}\right), A_{w}=\left(\begin{array}{cc}
0 & \psi \\
\varepsilon \bar{\psi} & 0
\end{array}\right), A_{z}=i \varepsilon\left(\begin{array}{cc}
-\psi \star \bar{\psi} & -\varepsilon \psi^{\prime} \\
\bar{\psi}^{\prime} & \bar{\psi} \star \psi
\end{array}\right)
$$

where $\varepsilon= \pm 1$. The case for $\varepsilon=-1$ coincides with results by Legaré [15].

We note that the gauge group must be $U(2)$ or $U(1,1)$ not $S U(2)$ or $S U(1,1)$, respectively on $\mathrm{NC}$ spaces because the matrix $A_{z}$ is not traceless. We prove that $U(1)$ part of the gauge group is crucial in this case also, and the choice by Legaré [15] is unique for the reduction.

Here we would like to point out that every $\mathrm{NC}$ conservation laws on $(1+1)$-dimensional space-time can be derived from NC ASDYM equations by a special reduction. ${ }^{3}$ Let us consider the following $G=S L(2, \mathbf{R})$ NC ASDYM equation, which is dimensionally reduced to 2-dimensional space-time with the coordinate $(t, x)=(z, w)$ and a fixed gauge $A_{\tilde{z}}=A_{\tilde{w}}=0$ :

$$
\partial_{x} A_{z}-\partial_{t} A_{w}+\left[A_{w}, A_{z}\right]_{\star}=0 .
$$

By taking further reduction

$$
A_{z}=\left(\begin{array}{cc}
0 & 0 \\
J & 0
\end{array}\right), A_{w}=\left(\begin{array}{ll}
0 & 0 \\
\sigma & 0
\end{array}\right)
$$

we get an arbitrary conservation law

$$
\partial_{t} \sigma=\partial_{x} J
$$

because $\sigma$ and $J$ can be taken arbitrarily. For example, $\sigma=4 u, J=u^{\prime \prime}+3 u \star u$ yields the NC KdV equation. (cf. [15].)

\section{NC $N$-wave, DS and KP Equations from ASDYM}

In this section, we discuss reductions of NC ASDYM equations where the components of gauge fields are elements of an infinite dimensional Lie algebra of formal matrix differential operators in an auxiliary variable $\partial_{y}$ :

$$
g=\left\{a_{0}+a_{1} \partial_{y}+a_{2} \partial_{y}^{2}\right\}
$$

\footnotetext{
${ }^{3}$ The derivation in this paragraph is trivial, because the gauge fields $A_{z}$ and $A_{w}$ in Eq. (3.16) can be set to zero via the following gauge transformation: $A_{z, w} \rightarrow g^{-1} A_{w, z} g+g^{-1} \partial_{z, w} g$, where $g=\left(\begin{array}{ll}1 & 0 \\ h & 1\end{array}\right)$ and $h(t, x)=-\int^{x} \sigma(t, y) d y$. This is an expected result. (The author thanks L. Mason for commenting on this point, during stay at Oxford supported by the Yamada Science Foundation.)
} 
where $a_{0}, a_{1}, a_{2}$ belong to a ring of $N \times N$ matrix functions of $y$. Here we follow the convention in [51].

- $\mathrm{NC} N$-wave equation

Let us suppose that all gauge fields depend on $t=z$ and $x=w$ only, which is a simple dimensional reduction. The linear system (2.3) is reduced as follows :

$$
\partial_{x} \Psi=\left(A_{w}+\zeta A_{\tilde{z}}\right) \star \Psi, \quad \partial_{t} \Psi=\left(A_{z}+\zeta A_{\tilde{w}}\right) \star \Psi
$$

where $\Psi$ is a function of $x, y, t$ and $\zeta$. Now let us take further reduction condition as $A_{w}=U+A \partial_{y}, A_{z}=V+B \partial_{y}, A_{\tilde{z}}=A_{\tilde{w}}=0$, where $A, B$ are constant, commuting $N \times N$ matrices: $[A, B]=0$. By taking $\Psi=\Phi e^{-\zeta y}$, we get a reduced linear system:

$$
\partial_{x} \Phi=\left(U+A \partial_{y}\right) \star \Phi, \quad \partial_{t} \Phi=\left(V+B \partial_{y}\right) \star \Phi .
$$

The compatible condition for the reduced linear system gives rise to a reduced ASDYM equation:

$$
U_{t}-V_{x}+[U, V]_{\star}+A \star V_{y}-B \star U_{y}=0, \quad[A, V]_{\star}=[B, U]_{\star},
$$

where $U_{t}:=\partial U / \partial t, V_{x}:=\partial V / \partial x$ etc. This results a non-linear equation:

$$
\left[A, Q_{t}\right]_{\star}-\left[B, Q_{x}\right]_{\star}+\left[[A, Q]_{\star},[B, Q]_{\star}\right]_{\star}+A \star\left[B, Q_{y}\right]_{\star}-B \star\left[A, Q_{y}\right]_{\star}=0,
$$

where $U=[A, Q]_{\star}, V=[B, Q]_{\star}$.

If we take $A, B$ as diagonal matrices: $A_{i j}=a_{i} \delta_{i j}, B_{i j}=b_{i} \delta_{i j}$, we get the $\mathrm{NC}(2+1)$ dimensional $N$-wave equation which is new:

$$
\frac{\partial U_{i j}}{\partial t}=\lambda_{i j} \frac{\partial U_{i j}}{\partial x}-\mu_{i j} \frac{\partial U_{i j}}{\partial y}+\sum_{k=1}^{N}\left(\lambda_{i k}-\lambda_{k j}\right) U_{i k} U_{k j}
$$

where $\lambda_{i j}:=\left(b_{i}-b_{j}\right) /\left(a_{i}-a_{j}\right), \mu_{i j}:=a_{i} \lambda_{i j}-b_{i}, \quad(i \neq j)$. In the commutative limit, this equation is reduced to the $(2+1)$-dimensional $N$-wave equation. It is interesting to study whether $\mathrm{NC} N$-wave equation still admits a configuration of $N$ interacting waves as commutative case [57].

- NC Davey-Stewartson equation

From now on, we assume $N=2$ and that all gauge fields depend on $t=z$ and $x=w+\tilde{w}$ only, which is another dimensional reduction. The linear system (2.3) is reduced as follows:

$$
\partial_{x} \Psi=\left(A_{w}+\zeta A_{\tilde{z}}\right) \star \Psi, \quad \partial_{t} \Psi=\left(A_{z}+\zeta\left(A_{w}+A_{\tilde{w}}\right)+\zeta^{2} A_{\tilde{z}}\right) \star \Psi
$$


where the coefficient matrices depend on $x, y$ and $t$. Now let us take $\Psi=\Phi e^{-\zeta y}$ and further reduction condition: $A_{w}=U+A \partial_{y}, A_{z}=V+\tilde{U} \partial_{y}+A \partial_{y}^{2}, A_{\tilde{z}}=A, A_{\tilde{w}}=$ $\tilde{U}-U+A \partial_{y}$, we get a reduced linear system:

$$
\partial_{x} \Phi=\left(U+A \partial_{y}\right) \star \Phi, \quad \partial_{t} \Phi=\left(V+\tilde{U} \partial_{y}+A \partial_{y}^{2}\right) \star \Phi .
$$

By taking further reduction condition:

$$
A=\kappa\left(\begin{array}{cc}
1 & 0 \\
0 & -1
\end{array}\right), U=\tilde{U}=\left(\begin{array}{cc}
0 & q \\
r & 0
\end{array}\right), V=\frac{1}{2 \kappa}\left(\begin{array}{cc}
R_{1} & \left(\partial_{x}+\kappa \partial_{y}\right) q \\
-\left(\partial_{x}-\kappa \partial_{y}\right) r & R_{2}
\end{array}\right),
$$

the compatibility condition yields a reduced ASDYM equation which is the NC Davey-Stewartson system:

$$
\begin{aligned}
& 2 \kappa \dot{q}=\left(\partial_{x}^{2}+\kappa^{2} \partial_{y}^{2}\right) q+R_{1} \star q-q \star R_{2}, \\
& 2 \kappa \dot{r}=-\left(\partial_{x}^{2}+\kappa^{2} \partial_{y}^{2}\right) r+R_{2} \star r-r \star R_{1},
\end{aligned}
$$

and

$$
\begin{aligned}
& \left(\partial_{x}-\kappa \partial_{y}\right) R_{1}=-\left(\partial_{x}+\kappa \partial_{y}\right)(q \star r), \\
& \left(\partial_{x}+\kappa \partial_{y}\right) R_{2}=\left(\partial_{x}-\kappa \partial_{y}\right)(r \star q) .
\end{aligned}
$$

These are also new equations. By taking a dimensional reduction $\partial_{y}=0$, this discussion is reduced to that of NC NLS equation with $R_{1}=-q \star r, R_{2}=r \star q, q=$ $\psi, r=\tilde{\psi}$.

- NC KP equation

NC KP equation can be derived from NC ASDYM equation in similar discussion to NC DS equation. Let us start from the reduced system (4.8), and take another further reduction on the gauge fields as follows:

$$
A=\left(\begin{array}{ll}
0 & 0 \\
1 & 0
\end{array}\right), U=\left(\begin{array}{ll}
0 & 1 \\
u & 0
\end{array}\right), \tilde{U}=\left(\begin{array}{cc}
0 & 1 \\
u / 2 & 0
\end{array}\right), V=\frac{1}{4}\left(\begin{array}{cc}
\alpha+u_{x} & -2 u \\
u_{x x}-2 u \star u+u_{y} & \alpha-u_{x}
\end{array}\right) .
$$

Then the compatibility condition gives rise to a NC reduced ASDYM equation:

$$
\left(\begin{array}{cc}
-\alpha_{x}-3 u_{y} & 0 \\
4 u_{t}-u_{x x x}+3 u_{x} \star u+3 u \star u_{x}+\alpha_{y}+[u, \alpha]_{\star} & -\alpha_{x}-3 u_{y}
\end{array}\right)=0 .
$$

Hence we get $\alpha=-3 \partial_{x}^{-1} u_{y}:=-3 \int^{x} u_{y}\left(x^{\prime}\right) d x^{\prime}$, and the NC KP equation [6, 17]:

$$
u_{t}=\frac{1}{4}\left(u_{x x x}-3\left(u_{x} \star u+u \star u_{x}\right)+3 \partial_{x}^{-1} u_{y y}+3\left[u, \partial_{x}^{-1} u_{y}\right]_{\star}\right) \text {. }
$$

If we neglect $y$-dependence, the present discussion gives rise to another reduction to NC KdV equation from ASDYM equation with $G=S L(2, \mathbf{R})$, which is reduced to discussion by Bakas and Depireux [58] in the commutative limit.

Reduction of NC ASDYM hierarchies to NC integrable hierarchies would be possible in similar ways. The detailed discussion would be reported somewhere. 


\section{Conclusion and Discussion}

In the present letter, we proved that various $\mathrm{NC}$ integrable equations can be derived from NC ASDYM equations in the split signature, which include NC versions of KdV, NLS, $N$-wave, DS and KP equations. $U(1)$ part of gauge groups for the original Yang-Mills equations play crucial roles in NC extension of the Mason-Sparling's beautiful result. Existence of these reductions guarantees the lower-dimensional integrable equations actually have the corresponding physical situations, such as, reduced D0-D4 D-brane systems. Then, Analysis of exact NC soliton solutions could be applied to that of D-brane dynamics in the special reduced situations.

In reductions of NC ASDYM equations, we mainly should take the split signature. NC Yang-Mills theories with the split signature can be embedded [54] in $\mathrm{N}=2$ string theories [59]. Simple reductions of them and relation to the string theories have been studied intensively [18, 22, 23, 29, 47. This guarantees that NC integrable equations would have physical meanings and might lead to various successful applications to the corresponding D-brane dynamics and so on.

One of the next steps is to study supersymmetric extension (e.g [15, 33]) of these reductions of $\mathrm{NC}$ ASDYM equations in order to clarify what reductions are realized in the framework of D-brane systems in $\mathrm{N}=2$ string theories. We can judge what reductions are better or worse from the viewpoint of the number of the preserved supersymmetry or stability of the corresponding D-brane configurations. This might exclude the special reductions to any conservation laws as we commented in the end of section 3 .

The BPS equations in some D-brane configurations would just correspond to NC integrable equations and the soliton solutions correspond to lower-dimensional D-branes. In these situations, we can expect that similar applications to D-brane dynamics would be possible, as in tachyon condensations. NC solitons are sometimes very easy to treat in infinite noncommutativity limit. The typical example is known as the GopakumarMinwalla-Strominger (GMS) soliton [60]. GMS-like solitons would exist in our situations in some limit and we could construct exact soliton solutions and analyze energy densities of them and fluctuation spectrum around them and so on. This would be a hint to reveal the corresponding D-brane configurations which might be new BPS states.

\section{Acknowledgments}

The author would like to thank H. Kanno, O. Lechtenfeld, L. Mason, S. Moriyama, F. Müller-Hoissen, A. Popov and K. Takasaki for fruitful discussions and useful comments. He is also grateful to O. Lechtenfeld, F. Müller-Hoissen and K. Lee for financial support and hospitality during stays at the Institute for Theoretical Physics, University of 
Hannover, and the Max Planck Institute for dynamics and self-organization, Göttingen, and the Korea Institute for Advanced Study, on March 2005. This work was partially supported by the Daiko Foundation (\#9095).

\section{References}

[1] J. A. Harvey, "Komaba lectures on noncommutative solitons and D-branes," hep-th/0102076; M. R. Douglas and N. A. Nekrasov, Rev. Mod. Phys. 73 (2002) 977 hep-th/0106048; R. J. Szabo, Phys. Rept. 378 (2003) 207 hep-th/0109162; C. S. Chu, "Non-commutative geometry from strings," hep-th/0502167 and references therein.

[2] A. Sen, "Tachyon dynamics in open string theory," hep-th/0410103 and references therein.

[3] K. Furuuchi, "Topological charge of U(1) instantons on noncommutative $\mathbf{R}^{4}$," hep-th/0010006; N. A. Nekrasov, "Trieste lectures on solitons in noncommutative gauge theories," hep-th/0011095; A. Konechny and A. Schwarz, Phys. Rept. 360 (2002) 353 hep-th/0107251; M. Hamanaka, "Noncommutative solitons and D-branes," Ph. D thesis, hep-th/0303256, F. A. Schaposnik, Braz. J. Phys. 34 (2004) 1349 hep-th/0310202; O. Lechtenfeld, Fortsch. Phys. 52 (2004) 596 hep-th/0401158 and references therein.

[4] M. Hamanaka, "Noncommutative solitons and integrable systems," hep-th/0504001;

L. Tamassia, "Noncommutative supersymmetric / integrable models and string theory," Ph. D thesis, hep-th/0506064.

[5] T. Curtright, D. Fairlie and C. K. Zachos, Phys. Lett. B 405 (1997) 37 hep-th/9704037; C. K. Zachos, D. Fairlie and T. Curtright, "Matrix membranes and integrability," hep-th/9709042.

[6] B. Kupershmidt, KP or mKP : noncommutative mathematics of Lagrangian, Hamiltonian, and integrable systems (AMS, 2000) [ISBN/0821814001].

[7] A. Dimakis and F. Müller-Hoissen, Int. J. Mod. Phys. B 14 (2000) 2455 hep-th/0006005; Lett. Math. Phys. 54 (2000) 123 hep-th/0007160; J. Phys. A 34 (2001) 9163 nlin.si/0104071.

[8] A. Dimakis and F. Müller-Hoissen, "A noncommutative version of the nonlinear Schroedinger equation," hep-th/0007015; Czech. J. Phys. 51 (2001) 1285. 
[9] A. Dimakis and F. Müller-Hoissen, Phys. Lett. A 278 (2000) 139 hep-th/0007074.

[10] A. Dimakis and F. Müller-Hoissen, J. Phys. A 34 (2001) 2571 nlin.si/0008016.

[11] A. Kapustin, A. Kuznetsov and D. Orlov, Commun. Math. Phys. 221 (2001) 385 hep-th/0002193.

[12] K. Takasaki, J. Geom. Phys. 37 (2001) 291 hep-th/0005194.

[13] D. J. Gross and N. A. Nekrasov, JHEP 0007 (2000) 034 hep-th/0005204.

[14] N. A. Nekrasov, Commun. Math. Phys. 241 (2003) 143 hep-th/0010017.

[15] M. Legare, "Noncommutative generalized NS and super matrix KdV systems from a noncommutative version of (anti-)self-dual Yang-Mills equations," hep-th/0012077; J. Phys. A 35 (2002) 5489.

[16] O. Lechtenfeld, A. D. Popov and B. Spendig, JHEP 0106 (2001) 011 hep-th/0103196.

[17] L. D. Paniak, "Exact noncommutative KP and KdV multi-solitons," hep-th/0105185.

[18] O. Lechtenfeld and A. D. Popov, JHEP 0111 (2001) 040 hep-th/0106213; Phys. Lett. B 523 (2001) 178 hep-th/0108118.

[19] K. C. Hannabuss, Lett. Math. Phys. 58 (2001) 153 hep-th/0108228.

[20] O. Lechtenfeld and A. D. Popov, JHEP 0203 (2002) 040 hep-th/0109209.

[21] M. T. Grisaru and S. Penati, Nucl. Phys. B 655 (2003) 250 hep-th/0112246.

[22] S. Bieling, J. Phys. A 35 (2002) 6281 hep-th/0203269|.

[23] M. Wolf, JHEP 0206 (2002) 055 hep-th/0204185.

[24] K. Toda, "Extensions of soliton equations to non-commutative $(2+1)$ dimensions," Proceedings of workshop on integrable theories, solitons and duality, Sao Paulo, Brazil, 1-6 July 2002 [JHEP PRHEP-unesp2002/038].

[25] Z. Horváth, O. Lechtenfeld and M. Wolf, JHEP 0212 (2002) 060 hep-th/0211041.

[26] K. Furuta, T. Inami and M. Yamamoto, "Topics in nonlinear sigma models in D = 3 ," hep-th/0211129.

[27] M. Hamanaka and K. Toda, Phys. Lett. A 316 (2003) 77 hep-th/0211148. 
[28] I. Cabrera-Carnero and M. Moriconi, Nucl. Phys. B 673 (2003) 437 hep-th/0211193; hep-th/0303168.

[29] M. Ihl and S. Uhlmann, Int. J. Mod. Phys. B 18 (2003) 4889 hep-th/0211263.

[30] M. Hamanaka and K. Toda, J. Phys. A 36 (2003) 11981 hep-th/0301213.

[31] L. Martina and O. K. Pashaev, "Burgers' equation in non-commutative space-time," hep-th/0302055.

[32] O. Lechtenfeld and A. D. Popov, JHEP 0401 (2004) 069 hep-th/0306263.

[33] H. Nishino and S. Rajpoot, Phys. Lett. B 572 (2003) 91 hep-th/0306290.

[34] N. Wang and M. Wadati, J. Phys. Soc. Jap. 72 (2003) 1366; J. Phys. Soc. Jap. 72 (2003) 1881; J. Phys. Soc. Jap. 72 (2003) 3055. J. Phys. Soc. Jap. 73 (2004) 1689.

[35] A. Zuevsky, J. Phys. A 37 (2004) 537.

[36] M. Hamanaka and K. Toda, Proc. Inst. Math. NAS Ukraine (2004) 404 hep-th/0309265.

[37] M. T. Grisaru, L. Mazzanti, S. Penati and L. Tamassia, JHEP 0404 (2004) 057 hep-th/0310214.

[38] M. Hamanaka, J. Math. Phys. 46 (2005) 052701 hep-th/0311206.

[39] A. Dimakis and F. Müller-Hoissen, J. Phys. A 37 (2004) 4069 hep-th/0401142; J. Phys. A 37 (2004) 10899 hep-th/0406112 ; "Extension of Moyal-deformed hierarchies of soliton equations," nlin.si/0408023.

[40] K. Shigechi, M. Wadati and N. Wang, Nucl. Phys. B 706 (2005) 518 hep-th/0404249.

[41] K. M. Lee, JHEP 0408 (2004) 054 hep-th/0405244.

[42] O. Lechtenfeld, L. Mazzanti, S. Penati, A. D. Popov and L. Tamassia, hep-th/0406065.

[43] M. Sakakibara, J. Phys. A 37 (2004) L599 nlin.si/0408002.

[44] A. Dimakis and F. Müller-Hoissen, J. Phys. A 38 (2005) 5453 nlin.si/0501003; "Algebraic identities associated with KP and AKNS hierarchies," math-ph/0507024.

[45] H. Blas, H. L. Carrion and M. Rojas, JHEP 0503 (2005) 037 hep-th/0502051. 
[46] I. Cabrera-Carnero, "Abelian Toda field theories on the noncommutative plane," hep-th/0503147.

[47] C. S. Chu and O. Lechtenfeld, "Time-space noncommutative abelian solitons," hep-th/0507062.

[48] R. S. Ward, Phil. Trans. Roy. Soc. Lond. A 315 (1985) 451; "Multidimensional integrable systems," Lect. Notes Phys. 280 (Springer, 1986) 106 "Integrable systems in twistor theory," in Twistors in Mathematics and Physics (Cambridge UP, 1990) 246 [ISBN/0-521-39783-9].

[49] L. J. Mason and N. M. Woodhouse, Integrability, Self-Duality, and Twistor Theory (Oxford UP, 1996) [ISBN/0-19-853498-1].

[50] M. J. Ablowitz and P. A. Clarkson, Solitons, Nonlinear Evolution Equations and Inverse Scattering (Cambridge UP, 1991) Chapter 6.5 [ISBN/0-521-38730-2].

[51] M. J. Ablowitz, S. Chakravarty and L. A. Takhtajan, Commun. Math. Phys. 158 (1993) 289;

[52] T. A. Ivanova and A. D. Popov, Theor. Math. Phys. 102 (1995) 280 [Teor. Mat. Fiz. 102 (1995) 384]; S. Chakravarty, S. L. Kent and E. T. Newman, J. Math. Phys. 36 (1995) 763; M. J. Ablowitz, S. Chakravarty and R. G. Halburd, J. Math. Phys. 44 (2003) 3147.

[53] L. J. Mason and G. A. J. Sparling, Phys. Lett. A 137 (1989) 29.

[54] O. Lechtenfeld, A. D. Popov and B. Spendig, Phys. Lett. B 507 (2001) 317 hep-th/0012200.

[55] J. E. Moyal, Proc. Cambridge Phil. Soc. 45 (1949) 99; H. J. Groenewold, Physica 12 (1946) 405.

[56] M. Dunajski and G. Sparling, Phys. Lett. A 343 (2005) 129 nlin.si/0503030.

[57] M. Ablowitz and R. Haberman, J. Math. Phys. 16 (1975) 2301.

[58] I. Bakas and D. A. Depireux, Mod. Phys. Lett. A 6 (1991) 399.

[59] H. Ooguri and C. Vafa, Mod. Phys. Lett. A 5 (1990) 1389; Nucl. Phys. B 361 (1991) 469; Nucl. Phys. B 367 (1991) 83.

[60] R. Gopakumar, S. Minwalla and A. Strominger, JHEP 0005 (2000) 020 hep-th/0003160. 